\title{
(6) OPEN ACCESS \\ Amyloid-related imaging abnormalities-haemosiderin (ARIA-H) in patients with Alzheimer's disease treated with bapineuzumab: a historical, prospective secondary analysis
}

\author{
H Michael Arrighi, ${ }^{1}$ Jerome Barakos, ${ }^{2,3}$ Frederik Barkhof, ${ }^{4,5}$ Donatella Tampieri, ${ }^{6}$ \\ Clifford Jack Jr, ${ }^{7}$ Denis Melançon, ${ }^{7}$ Kristen Morris, ${ }^{8,9}$ Nzeera Ketter, ${ }^{1}$ Enchi Liu, ${ }^{1}$ \\ H Robert Brashear ${ }^{1}$
}

- Additional material is published online only. To view please visit the journal online (http://dx.doi.org/10.1136/ jnnp-2014-309493).

For numbered affiliations see end of article.

\section{Correspondence to}

Dr H Michael Arrighi, Janssen Pharmaceuticals Research \& Development, 40 King Street, Mill Valley, South

San Francisco, CA 94941,

USA; marrighi@its.jnj.com

Received 28 September 2014 Revised 7 January 2015

Accepted 19 January 2015 Published Online First

10 February 2015

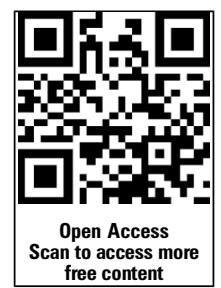

CrossMark

\footnotetext{
To cite: Arrighi $\mathrm{HM}_{\mathrm{t}}$ Barakos J, Barkhof $F_{\text {, et al. }}$. $J$ Neurol Neurosurg Psychiatry 2016;87: 106-112.
}

\section{ABSTRACT}

Background Amyloid-related imaging abnormalities due to haemosiderin deposition (ARIA-H) occur in patients with mild to moderate dementia due to Alzheimer's disease (AD) and have been reported with increased incidence in clinical trials of amyloid-lowering therapies under development for AD.

Objective Our objective was to explore the relationship between the incidences of ARIA-H during treatment with placebo and different doses of bapineuzumab, a humanised monoclonal antibody directed against amyloid $\beta$.

Methods Two neuroradiologists independently reviewed 2572 GRE/T2* MRI sequences from 262 participants in two phase two clinical trials of bapineuzumab and an open-label extension study. Readers were blinded to the participant's therapy, APOE $\varepsilon 4$ genotype and medical history.

Results Several risk factors for small ARIA-H $<10 \mathrm{~mm}$ (microhaemorrhages) were identified: APOE $\varepsilon 4$, bapineuzumab treatment, pre-existing small ARIA-H and use of antithrombotics. The HR $(95 \% \mathrm{Cl})$ for incident ARIA-H $<10 \mathrm{~mm}$ associated with the number of APOE $\varepsilon 4$ alleles was 11.9 (3.3 to 42.5$)$ for 2 versus no alleles and 3.5 (1.0 to 12.0) for 1 versus no allele. The HR for bapineuzumab therapy was $3.5(1.0$ to 12.0$)$; for the presence of baseline ARIA-H $<10 \mathrm{~mm}$, it was 3.5 (1.6 to 7.8), and for the use of antithrombotic agents it was 2.2 (1.0 to 4.8). The incidence rate for ARIA-H $<10 \mathrm{~mm}$ was elevated only in the initial 6 months of active treatment and declined after this interval to a rate similar to that observed in the group treated with placebo.

Conclusions ARIA-H represents a spectrum of MRI findings due to haemosiderin deposition that appears to be related to impaired vascular integrity. The increased risk for ARIA-H associated with APOE $\varepsilon 4$ allele frequency, pre-existing ARIA-H, treatment with bapineuzumab and use of antithrombotic agents provides additional support for this hypothesis of loss of integrity of cerebral vessels due to amyloid burden.

Trial registration NCT00112073 and NCT00606476.

\section{INTRODUCTION}

Cerebral microhaemorrhages (mHs), also known as microbleeds, are identified as small areas of hypointense signal on T2* MRIs, with an increasing prevalence with increasing age in the general population. ${ }^{1}{ }^{2}$ They are commonly observed in patients with Alzheimer's disease (AD) dementia with prevalence rates ranging from $16 \%$ to $32 \% .^{3-6}$ Cerebral $\mathrm{mHs}$ are more common in patients with $\mathrm{AD}$ dementia and individuals with mild cognitive impairment than in the general population, while being less common in persons with ischaemic or haemorrhagic stroke. ${ }^{7}$ Cerebral $\mathrm{mHs}$ in patients with $\mathrm{AD}$ appear to be related to amyloid deposition and share a similar pattern to that observed in cerebral amyloid angiopathy (CAA). ${ }^{5} 8$

Concerns raised by the US Food and Drug Administration regarding MRI abnormalities observed associated with amyloid-modifying therapy in patients with $\mathrm{AD}$ prompted the Alzheimer's Association to convene a work group. ${ }^{9}$ The work group coined the phrase ARIA (amyloid-related imaging abnormalities) to describe a spectrum of MRI findings that include sulcal effusion and parenchymal edema (ARIA-E) and haemosiderin deposition (ARIA-H). Specifically, ARIA-H refers to areas of hypointensity on gradient echo MRI that are believed to represent deposits of iron in the form of haemosiderin. ARIA represents a spectrum of changes including sulcal effusion and parenchymal edema (ARIA-E), and haemosiderin deposition (ARIA-H). Animal models indicate that anti-amyloid $\beta$ treatment removes vascular amyloid with a corresponding compromise of the integrity of the vascular wall and leakage of blood resulting in microhaemorrhages and haemosiderin deposition. ${ }^{10}$ This report summarises the ARIA-H findings from a review of MRI from three studies of immunotherapy in $A D$ dementia. ARIA-H was categorised as lesions on MRI $<10 \mathrm{~mm}$ and $\geq 10 \mathrm{~mm}$. The review was undertaken to describe the incidence of ARIA-H and explore potential associations and risk factors, in part to improve evaluations of subsequent studies of amyloid-modifying therapies.

\section{METHODS}

A centralised review was conducted on all MRIs performed during the completed phase 2 bapineuzumab clinical trials (study $201^{11}$ and study $202^{12}$ ) and the associated ongoing open-label extension study, study 251, prior to 1 February 2009. MRI 
had been performed as part of routine safety evaluation 6 weeks after each infusion following a protocol-specified series that included T2*/GRE sequences. The following procedures were adopted to ensure ARIA detection: (1) two neuroradiologists, blinded to all clinical data, retrospectively reviewed the scans with complete access to all MRIs and all time points for comparison; (2) all scans were read independently and in parallel by each reader and (3) differences between readings were then discussed and resolved by consensus. The numbers of ARIA-H lesions, small and large, were identified on each scan.

Studies 201 and 202 were multicentre, double-blinded, randomised, placebo-controlled, ascending-dose cohort trials of bapineuzumab in patients with mild to moderate AD. ${ }^{11}{ }^{12}$ Each lasted for 18 months and included six infusions 13 weeks apart. Study 251 was an extension study into which eligible study 201 participants were recruited. Participants in study 251 who previously received bapineuzumab in study 201 were assigned the same dose of bapineuzumab in study $251(\mathrm{n}=80)$, and those who previously received placebo in study $201(\mathrm{n}=79)$ received the dose level of active drug assigned to the cohort to which they were originally assigned. No participants from study 202 enrolled in study 251 . MRIs were performed prior to the first infusion and 6 weeks after each subsequent infusion in all studies. The bapineuzumab phase 2 trials were approved by each site's local institutional review board, and written informed consent was obtained from each participant (or legally authorised representative). All data from participants in studies 201 and 202, as well as from participants in study 251 until 31 January 2009, were reviewed for inclusion. Data from 23 participants were excluded because either a baseline or follow-up GRE MRI was missing or unsuitable for neuroradiological assessment. All patients met the inclusion and exclusion criteria for mild to moderate dementia due to $\mathrm{AD}$ as required by the clinical studies. ARIA-H findings were categorised by size, either less than $10 \mathrm{~mm}$ in the longest diameter or larger. In nearly all cases, ARIA-H $<10 \mathrm{~mm}$ are equivalent to $\mathrm{mHs}$ (microbleeds) identified on MRI; however, for precision, the term based on study criteria is used in this report. The number of ARIA-H $<10 \mathrm{~mm}$ were recorded and counted using the procedures outlined for the MARS (Microbleed Anatomical Rating Scale), which enumerates the total, definite and possible $\mathrm{mHs}$ within specified brain anatomical regions. ${ }^{13}$ In these analyses, the total count was used. For the scans where more than one ARIA-H was identified, the inter-rater concordance correlation coefficient for the total count of ARIA-H $<10 \mathrm{~mm}$ was 0.93. The $\kappa$ statistic for agreement on the presence or absence of ARIA-H was 0.89 .

White matter hyperintensities (WMH) were assessed using an adapted four-point scale: ${ }^{14} 0$ (none), 1 (focal lesions), 2 (beginning of confluent lesions) and 3 (diffuse involvement). For the risk analyses, WMH levels 0 and 1 were combined into a single category, and levels 2 and 3 were combined into a single category due to the limited number of scans in each of the 4 levels.

Two analytical groups were defined based on treatment with bapineuzumab, and to be included in the analysis set they had to have MRI and at least one post-infusion image. Of 223 patients treated with bapineuzumab, 201 were eligible for these analyses, and of 118 placebo subjects, 100 were eligible for these analyses. A subject who was on placebo and transitioned to bapineuzumab therapy during the open-label extension study would contribute observation time to the group, placebo and bapineuzumab. Participants classified with incident ARIA-H were identified if a subsequent MRI had a greater ARIA-H count than the ARIA-H count on the screening MRI.
For patients with ARIA-E, the investigator was queried if the patient was considered symptomatic or asymptomatic. A patient was considered symptomatic if one or more symptoms were considered related or possibly related to the study drug in the ARIA safety report completed by the investigator.

This study is a secondary analysis using a historical, prospective cohort design from a randomised clinical trial. Cox proportional hazard $(\mathrm{CPH})$ models were used to explore risk factors. SAS release V.9.2 was used for all statistical analyses. The CPH assumption was evaluated by examining $\log$, negative log plots, with no observed violations. The factors used in selecting the most parsimonious model followed a backward selection procedure using a minimum Bayesian Information Criterion with the criterion to remain in the model being a $\mathrm{p}$ value of $<0.10$.

\section{RESULTS}

Table 1 presents the demographics and baseline information for participants who received a placebo or an active drug. Of the 100 participants initially assigned to placebo, $63(63 \%)$ eventually transitioned to bapineuzumab treatment and thus contributed information to both the active and placebo groups. Overall, the two groups were fairly similar for most characteristics. On average, participants assigned to bapineuzumab were slightly older and had a slightly lower Mini-Mental State Examination (MMSE) score at baseline than those in the placebo group, which

Table 1 Subject demographics and baseline characteristics by treatment group

\begin{tabular}{|c|c|c|c|}
\hline & $\begin{array}{l}\text { Active } \\
\mathrm{n}=201\end{array}$ & $\begin{array}{l}\text { Placebo } \\
\mathrm{n}=100\end{array}$ & p Value* \\
\hline Women & $102(50.75 \%)$ & 59 (59.00\%) & 0.1763 \\
\hline White & $194(97.52 \%)$ & $96(96.00 \%)$ & 0.8217 \\
\hline \multicolumn{4}{|l|}{ APOE $\varepsilon 4$} \\
\hline None & $72(35.82 \%)$ & $29(29.00 \%)$ & 0.4751 \\
\hline 1 & $97(48.26 \%)$ & $52(52.00 \%)$ & \\
\hline 2 & $32(15.92 \%)$ & $19(19.00 \%)$ & \\
\hline Hypertensive & $56(27.86 \%)$ & $31(31.00 \%)$ & 0.5715 \\
\hline Antithrombotic use & $102(50.75 \%)$ & $40(40.00 \%)$ & 0.0786 \\
\hline $\begin{array}{l}\text { Hamilton Depression Rating } \\
\text { Scale score }>2\end{array}$ & $68(34.00 \%)$ & $39(39.00 \%)$ & 0.3941 \\
\hline Modified Hachinski score $>0.5$ & $93(46.27 \%)$ & $46(46.00 \%)$ & 0.9649 \\
\hline ARIA-E on baseline MR & $2(1.00 \%)$ & $0(0.00 \%)$ & 0.4452 \\
\hline ARIA-H $<10 \mathrm{~mm}$ on baseline MR & $20(9.95 \%)$ & $8(8.00 \%)$ & 0.5832 \\
\hline ARIA-H $\geq 10 \mathrm{~mm}$ on baseline MR & $5(2.49 \%)$ & $0(0.00 \%)$ & 0.1306 \\
\hline \multicolumn{4}{|l|}{ Age in years } \\
\hline Mean (SD) & $69.60(8.95)$ & $67.81(8.77)$ & 0.0997 \\
\hline Median & 71.0 & 69.0 & \\
\hline \multicolumn{4}{|l|}{ MMSE } \\
\hline Mean (SD) & $19.85(5.23)$ & $20.93(3.00)$ & 0.0568 \\
\hline Median & 20.0 & 21.0 & \\
\hline \multicolumn{4}{|l|}{ Dose } \\
\hline $0 \mathrm{mg} / \mathrm{kg}$ (placebo) & - & $100(100.00 \%)$ & \\
\hline $0.15 \mathrm{mg} / \mathrm{kg}$ & $42(20.90 \%)$ & - & \\
\hline $0.5 \mathrm{mg} / \mathrm{kg}$ & $57(28.36 \%)$ & - & \\
\hline $1.0 \mathrm{mg} / \mathrm{kg}$ & $62(30.85 \%)$ & - & \\
\hline $2.0 \mathrm{mg} / \mathrm{kg}$ & 40 (19.90\%) & - & \\
\hline
\end{tabular}

Hypertension is defined by the use of an antihypertensive therapy, systolic blood pressure greater than $140 \mathrm{~mm} \mathrm{Hg}$ or diastolic blood pressure greater than $90 \mathrm{~mm} \mathrm{Hg}$. ${ }^{*} \chi^{2}$ when categorical, Fisher's exact test when the expected number is less than 5 , $t$ test when continuous.

ARIA-E, amyloid-related imaging abnormalities-edema; ARIA-H, ARIA-haemosiderin; MMSE, Mini-Mental State Examination. 
includes placebo subjects who transitioned to active therapy after 18 months of follow-up. Overall, 54\% of participants were women with a mean age of 69 years, and the majority of participants were Caucasian. Thirty-four per cent of participants did not carry an APOE $\varepsilon 4$ allele, $29 \%$ were classified as hypertensive, and $47 \%$ were using an antithrombotic agent.

On baseline MRI, 29 participants had evidence of either ARIA-H $<10 \mathrm{~mm}(\mathrm{n}=28)$ or ARIA-E $(\mathrm{n}=2)$. Of the two participants with ARIA-E, one had evidence of ARIA-H $<10 \mathrm{~mm}$. Another five participants had ARIA-H $\geq 10 \mathrm{~mm}$ with no evidence of ARIA-H $<10 \mathrm{~mm}$ or ARIA-E. Prior to consensus, the inter-reader $\kappa$ was 0.76 with $94 \%$ agreement on the presence or absence of ARIA-E within individual participants.

\section{ARIA-H $<10 \mathrm{~mm}$}

Figure 1 plots the first occurrence of a numeric increase in the total number of ARIA-H $<10 \mathrm{~mm}$ by the scan date relative to active therapy. For placebo subjects who never received active therapy, the start date was set at -18 months. The figure conveys that the more ARIA-H $<10 \mathrm{~mm}$ appeared on active therapy than on placebo with some indication of a decrease in the number of incident ARIA-H $<10 \mathrm{~mm}$, the longer a patient remains on active therapy. Two bapineuzumab subjects had an increase in ARIA-H $<10 \mathrm{~mm}$ to more than 20 within 3 months after the first infusion with active therapy. One placebo subject had a count of 20 ARIA-H $<10 \mathrm{~mm}$ at baseline that gradually increased over time and after initiation of bapineuzumab, the ARIA-H count increased by one with a subsequent decrease by one and was considered an incident event according to the pre-specified definition.

The two bapineuzumab-treated patients with large increases in ARIA-H $<10 \mathrm{~mm}$ (from none to 21 , and from 7 to 20 ) were both assigned to the $2 \mathrm{mg} / \mathrm{kg}$ dose group. Both were APOE $\varepsilon 4$ carriers, one homozygous and one heterozygous. Additionally, both of these patients developed ARIA-E. The placebo subject who had a count of 25 ARIA-H $<10 \mathrm{~mm}$ at baseline was homozygous for APOE $\varepsilon 4$; this patient did not experience ARIA-E before or after transitioning to active therapy.

Three participants assigned to placebo exhibited an increase in the count of ARIA-H $<10 \mathrm{~mm}$. Two had a single incident ARIA-H, while the third participant had an increase of five over the period of the study. Among those assigned to bapineuzumab, 27 participants exhibited an increase in any scan following the baseline scan. The mean increase in count on this initial scan was 3.07 (SD 4.50), with a median increase of 1 , with the 75 th and 90th centiles at 4 and 7 , respectively. The maximum increase observed was 21.

Among the 26 MRIs that exhibited a decrease in the total ARIA-H $<10 \mathrm{~mm}$ count from the preceding scan, the mean decline was 1.69 (SD 1.52), with a median decline of 1 . Among the 23 MRIs with a decline while on active therapy, the mean decline was 1.74 (SD 1.60), with a median decline of 1 . Among the three MRIs with a decline while on placebo, the mean decline was 1.33 (SD 0.58), with a median decline of 1 .

Overall, the ARIA-H $<10 \mathrm{~nm}$ incidence rate was 7.3/100 person-years (95\% CI 5.0 to 10.3$)$. The incidence rate increased with the number of APOE $\varepsilon 4$ alleles, with rates of 1.9 (95\% CI 0.5 to 5.1 ), 7.1 (95\% CI 4.1 to 11.7 ) and 22.1 (95\% CI 12.4 to 36.7 ), for none, one and two alleles, respectively. The rate among those assigned to placebo was 2.6 (95\% CI 0.7 to 6.8 ) and the rate among those treated with bapineuzumab was 9.2 (95\% CI 6.2 to 13.2), which indicates an increased rate among those treated with bapineuzumab. However, no clear relationship of an increasing rate with increasing dose was observed.
The incidence rate of ARIA-H $<10 \mathrm{~mm}$ was also higher in participants in whom ARIA-H was present at baseline $(27.7 ; 95 \% \mathrm{CI}$ 13.7 to 50.5$)$ compared to those who did not have baseline ARIA-H (5.6; 95\% CI 3.5 to 8.3). Participants with WMH and antithrombotic use at baseline were both observed to have higher rates of incident ARIA-H compared to those without. Combinations of dose with APOE $\varepsilon 4$, ARIA-H $<10 \mathrm{~mm}$ and antithrombotic use did not reveal any relationships; however, many of the categories had only one or two events with corresponding wide CIs for the rate. Online supplementary table S1 presents the incidence rates for ARIA-H $<10 \mathrm{~mm}$ for each of the factors and online supplementary table S2 presents additional incidence rates for the combination of bapineuzumab treatment and selected factors.

Figure 2 shows the rate ratios (RR) for ARIA-H $<10$ for each of the potential risk factors. Hypertension status, gender and Hamilton depression score did not show any risk with RR near or less than one and wide CIs that extend above and below 1 . Bapineuzumab treatment, APOE e4 alleles and presence of small ARIA-H at baseline had increased RR; antithrombotic use had a slightly increased RR. Other factors had point estimates that are greater than 1.0 but with wide CIs.

Table 2 presents the proportional hazards for the reduced model, full model and several models evaluating different subsets. Figure 3 shows the final, reduced, multivariate proportional hazards model for incident ARIA-H $<10 \mathrm{~mm}$, including the factors of bapineuzumab, APOE $\varepsilon 4$ allele number, antithrombotic use and baseline ARIA-H $<10 \mathrm{~mm}$. While bapineuzumab appears to be associated with an increase in the HR, no association of an increasing HR with increasing dose was observed. The number of APOE $\varepsilon 4$ alleles was associated with an increasing HR. Antithrombotic use and the presence of baseline ARIA-H $<10 \mathrm{~mm}$ each increased the HR. Similar HRs were observed for the subset with baseline ARIA-H $<10 \mathrm{~mm}$ but not ARIA-E, and for those without either baseline ARIA-H $<10 \mathrm{~mm}$ or ARIA-E. For the subset with baseline ARIA-H but not ARIA-E, the CIs were substantially increased compared to the other models, indicating instability of the estimates, consistent with the small number of cases. Among those dosed, the risk for ARIA-H did not consistently increase with increasing dose.

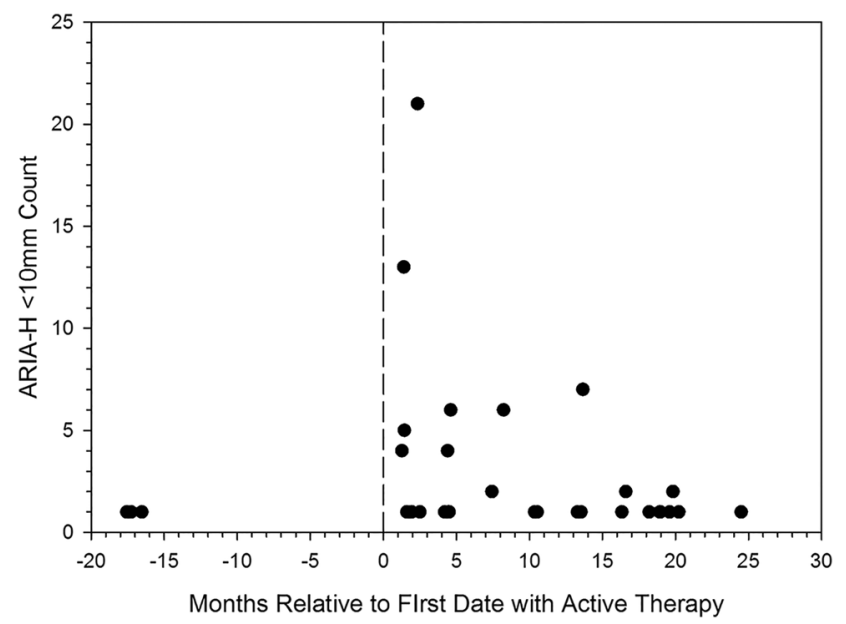

Figure 1 Total count on initial scan with a change from baseline in the number of amyloid-related imaging abnormalities-haemosiderin (ARIA-H) $<10 \mathrm{~mm}$, plotted by the amount of change relative to the date of first treatment with active therapy. For placebo subjects who did not transition to active therapy, the first placebo infusion date was set at month -18 . Each participant only appears once. 


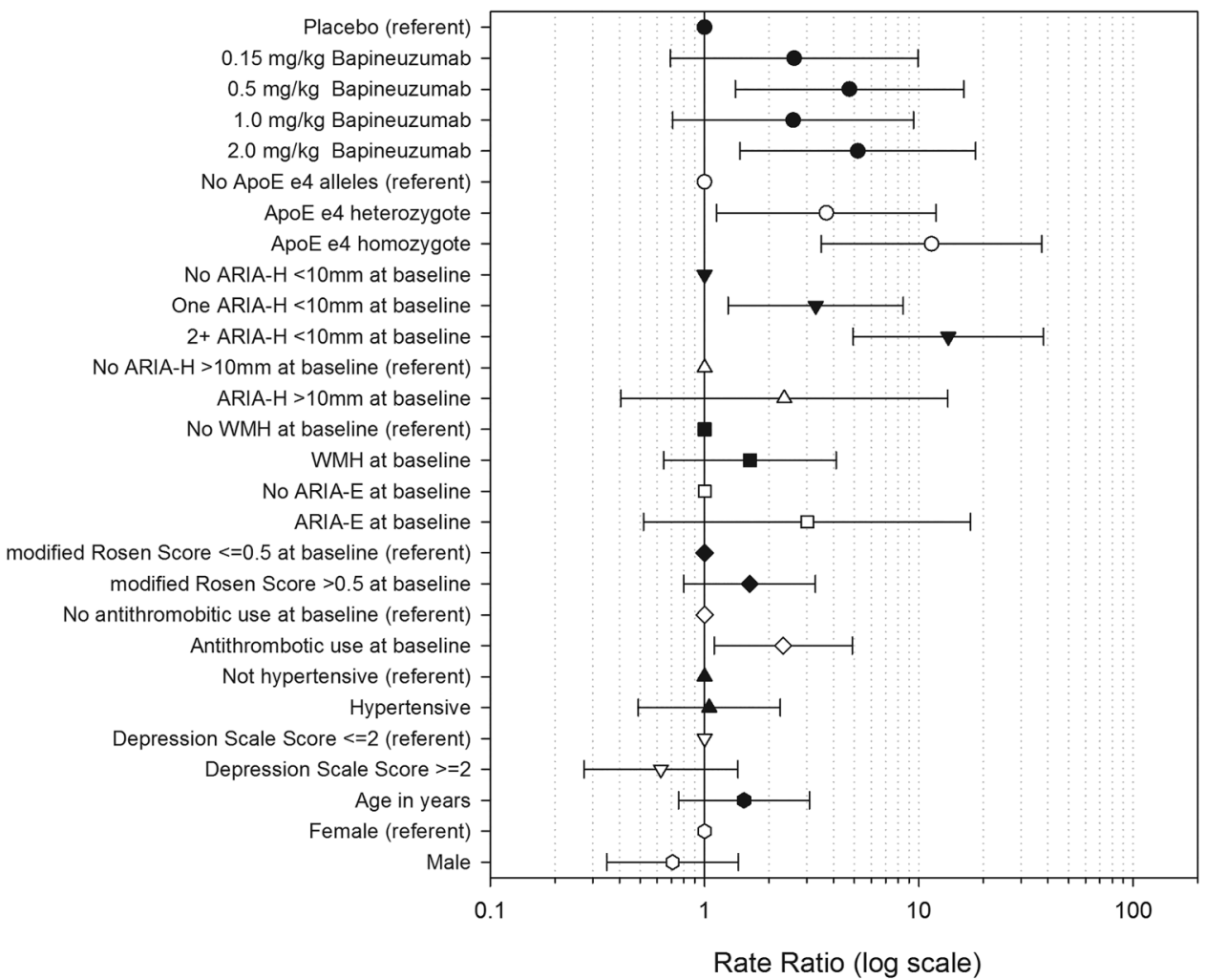

Figure 2 Crude rate ratios for incident amyloid-related imaging abnormalities-haemosiderin (ARIA-H) $<10$ mm. Hypertension is defined by the use of an antihypertensive therapy, systolic blood pressure greater than $140 \mathrm{~mm} \mathrm{Hg}$ or diastolic blood pressure greater than $90 \mathrm{~mm} \mathrm{Hg}$.

The incidence rates for ARIA-H $<10 \mathrm{~mm}$ within and after 9 months from first exposure to bapineuzumab are presented in table 3, along with the corresponding incidence rates for those initially assigned to placebo. The incidence rate during the first 9 months following initiation of therapy was 12.2 (95\% CI 7.6 to 19.9) per 100 person-years; afterwards, the rate was 6.8 per 100 person-years $95 \%$ CI (3.8 to 11.7). The overall rate among those assigned to placebo was $2.6 / 100$ person-years $95 \%$ CI (0.9 to 7.2).

Analysis of the joint incidence of ARIA-H $<10 \mathrm{~mm}$ and ARIA-E demonstrated that the two events occurred more commonly in the same participant than would be expected by the individual rates. The joint occurrence of ARIA- $\mathrm{H}<10 \mathrm{~mm}$ and ARIA-E was an incidence rate of 5.35 (95\% CI 3.24 to 8.38) per 100 person-years, which was greater than that predicted (3.45), if the individual event rates were independent (for ARIA-H $<10 \mathrm{~mm}, 9.22$ (95\% CI 6.21 to 13.21 ) and for ARIA-E, 11.80 (95\% CI 8.32 to 16.29$)$ ). The observed number of individuals with both events was 17 , with an observed-to-expected ratio of 4.92 (95\% CI 2.86 to 7.87 ), where the expected rate was based on the assumption of independence.

The co-location of incident ARIA-H $<10 \mathrm{~mm}$ and ARIA-E was based on nine regions. Of 17 participants who had both incident ARIA-H $<10 \mathrm{~mm}$ and ARIA-E, 15 participants had some events in the same region. Under the assumption of independence, 12.4 participants were estimated to have the two events in the same location by chance alone; the expectation by chance considers the number of affected regions with either ARIA-H $<10 \mathrm{~mm}$ or ARIA-E. The observed (17.0)-to-expected by change (12.4) ratio was 1.21 (95\% CI 0.68 to 1.99 ).

The CCC (correlation correspondence coefficient) for the total ARIA-H $<10 \mathrm{~mm}$ count was 0.93 (95\% CI 0.91 to 0.95 ). When ARIA-H $<10 \mathrm{~mm}$ was classified as either possible or definite, the CCC for definite ARIA-H $<10 \mathrm{~mm}$ was 0.85 (95\% CI
0.80 to 0.89$)$ and for possible ARIA-H $<10 \mathrm{~mm}, 0.83$ (95\% CI 0.78 to 0.88$)$.

After identification of an ARIA-E case, the investigator was queried whether the patient was considered to have had symptoms associated with the ARIA-E event. Among those participants with ARIA-E who were not symptomatic, the mean ARIA-H $<10 \mathrm{~mm}$ count increase from baseline was $1.22(95 \%$ CI 0.44 to 2.0); among those whose ARIA-E was considered symptomatic, the mean ARIA-H $<10 \mathrm{~mm}$ count increase from baseline was 5.67 (95\% CI -3.92 to 15.25$)$. Among those with symptomatic ARIA-E, 2 of the $6(33 \%)$ cases exhibited an increase in the ARIA-H $<10 \mathrm{~mm}$ number and both increases were large, 13 and 21. Of those not symptomatic due to ARIA-E, 13 of the 27 (48\%) exhibited an increase in the ARIA-H $<10 \mathrm{~mm}$ count, with the largest increase of 7 .

Figure 4 shows representative T2\%/GRE MRIs from a study participant who had no ARIA-H at baseline and developed incident ARIA-H associated with ARIA-E after receiving bapineuzumab, followed by gradual resolution of ARIA.

\section{ARIA-H >10 mm}

Three participants had new onset ARIA-H $\geq 10 \mathrm{~mm}$, consistent with superficial siderosis. One participant was on placebo and two were assigned to bapineuzumab, both at the $0.5 \mathrm{mg} / \mathrm{kg}$ dose. Two were homozygous for the APOE $\varepsilon 4$ allele, both assigned to bapineuzumab. The third case was a non-carrier for APOE عe4 assigned to placebo. None of the three participants had ARIA-H at baseline, either $<10$ or $\geq 10 \mathrm{~mm}$.

\section{Haemorrhagic stroke}

One cerebral haemorrhage was reported in a bapineuzumabtreated patient. Based on the rate of $3.3 / 1000$ person-years reported by Tolappanen et $a l,{ }^{15}$ for the 410 person-years of 
Table 2 ARIA-H $<10 \mathrm{~mm}$ proportional hazards models, HRs and 95\% Cls

\begin{tabular}{|c|c|c|c|c|c|c|c|c|c|}
\hline & \multicolumn{3}{|c|}{ Reduced model (1) } & \multicolumn{3}{|c|}{ Reduced model (2) } & \multicolumn{3}{|c|}{ Full model } \\
\hline & \multirow[b]{2}{*}{ HR } & \multicolumn{2}{|c|}{$95 \% \mathrm{Cl}$} & \multirow[b]{2}{*}{ HR } & \multicolumn{2}{|c|}{$95 \% \mathrm{Cl}$} & \multirow[b]{2}{*}{ HR } & \multicolumn{2}{|c|}{$95 \% \mathrm{Cl}$} \\
\hline & & LL & UL & & LL & UL & & LL & UL \\
\hline Placebo (referent) & 1.00 & & & 1.00 & & & 1.00 & & \\
\hline Dose $0.15 \mathrm{mg} / \mathrm{kg}$ & 2.78 & 0.64 & 12.16 & & & & 3.66 & 0.95 & 14.16 \\
\hline Dose $0.5 \mathrm{mg} / \mathrm{kg}$ & 4.13 & 1.09 & 15.74 & & & & 2.64 & 0.62 & 11.20 \\
\hline Dose $1.0 \mathrm{mg} / \mathrm{kg}$ & 2.33 & 0.57 & 9.47 & & & & 7.04 & 1.62 & 30.65 \\
\hline Dose $2.0 \mathrm{mg} / \mathrm{kg}$ & 6.19 & 1.56 & 24.52 & & & & 2.46 & 0.50 & 12.22 \\
\hline Active & & & & 3.54 & 1.05 & 11.96 & & & \\
\hline No APOE ع4 allele (referent) & 1.00 & & & 1.00 & & & 1.00 & & \\
\hline APOE $\varepsilon 41$ vs none & 4.10 & 1.16 & 14.53 & 3.63 & 1.04 & 12.70 & 4.16 & 1.09 & 15.91 \\
\hline APOE $\varepsilon 42$ vs none & 12.79 & 3.58 & 45.76 & 11.89 & 3.33 & 42.50 & 14.79 & 3.92 & 55.74 \\
\hline No antithrombotic use (referent) & 1.00 & & & 1.00 & & & 1.00 & & \\
\hline Antithrombotic use & 2.20 & 0.99 & 4.89 & 2.19 & 1.00 & 4.76 & 2.11 & 0.86 & 5.19 \\
\hline No ARIA-H $<10 \mathrm{~mm}$ at baseline (referent) & 1.00 & & & 1.00 & & & 1.00 & & \\
\hline ARIA-H $<10 \mathrm{~mm}$ versus none & 3.58 & 1.59 & 8.06 & 3.46 & 1.56 & 7.67 & 3.98 & 1.53 & 10.31 \\
\hline Hamilton Psychiatric Rating Scale for Depression score $\leq 2$ at baseline (referent) & & & & & & & 1.0 & & \\
\hline Hamilton Psychiatric Rating Scale for Depression score $>2$ & & & & & & & 0.71 & 0.27 & 1.88 \\
\hline Modified Hachinski score $\leq 0.5$ at baseline (referent) & & & & & & & 1.00 & & \\
\hline Modified Hachinski score $>0.5$ & & & & & & & 1.69 & 0.75 & 3.82 \\
\hline Male (referent) & & & & & & & 1.00 & & \\
\hline Female & & & & & & & 0.60 & 0.26 & 1.37 \\
\hline White (referent) & & & & & & & 1.00 & & \\
\hline Non-white & & & & & & & 0.31 & 0.06 & 1.63 \\
\hline Age (in year) & & & & & & & 1.00 & 0.95 & 1.05 \\
\hline MMSE score & & & & & & & 0.97 & 0.88 & 1.07 \\
\hline Not hypertensive (referent) & & & & & & & 1.00 & & \\
\hline Hypertensive & & & & & & & 0.64 & 0.26 & 1.59 \\
\hline White Matter Hyperintensity Scale score $(0,1)$ (referent) & & & & & & & 1.00 & & \\
\hline White Matter Hyperintensity Scale score $(2,3)$ & & & & & & & 1.83 & 0.63 & 5.37 \\
\hline No ARIA-E at baseline (referent) & & & & & & & 1.00 & & \\
\hline ARIA-E at baseline & & & & & & & 0.94 & 0.09 & 9.82 \\
\hline
\end{tabular}

exposure to bapineuzumab, 1.37 events of cerebral haemorrhage would be expected with an observed-to-expected ratio of 0.73 (95\% CI (0.02 to 4.06), based on the Poisson distribution.

\section{DISCUSSION}

APOE $\varepsilon 4$, bapineuzumab treatment and baseline ARIA-H $<10 \mathrm{~mm}$ (mHs) were the most robust risk factors for incident ARIA-H $<10 \mathrm{~mm}$. Homozygosity for APOE $\varepsilon 4$ exhibited the greatest risk. The high incidence rate in the $2 \mathrm{mg} / \mathrm{kg}$ arm contributed to the increased risk associated with bapineuzumab, although overall there was no clear evidence of dose effect. In the presence of baseline $\mathrm{mHs}$, incidence rates on all doses increased and obscured any differences between doses. Cumulative incidence rates for doses less than $2 \mathrm{mg} / \mathrm{kg}$ were similar to placebo. Antithrombotic use was less robustly associated with risk of ARIA-H. The incidence rate for ARIA $<10 \mathrm{~mm}$ associated with bapineuzumab demonstrated a clear temporal pattern. The rate increased in the 9 months after initiation of bapineuzumab and then subsequently returned to close to the placebo rate. This indicates a potential reduction of risk for bapineuzumab-associated ARIA-H after a period of initial exposure to the drug. A similar pattern was noted for ARIA-E during bapineuzumab exposure with initial increased incidence followed by decreasing risk. ${ }^{16}$ Further evaluation is needed to explore the relationship of the number of ARIA-H $<10 \mathrm{~mm}$.

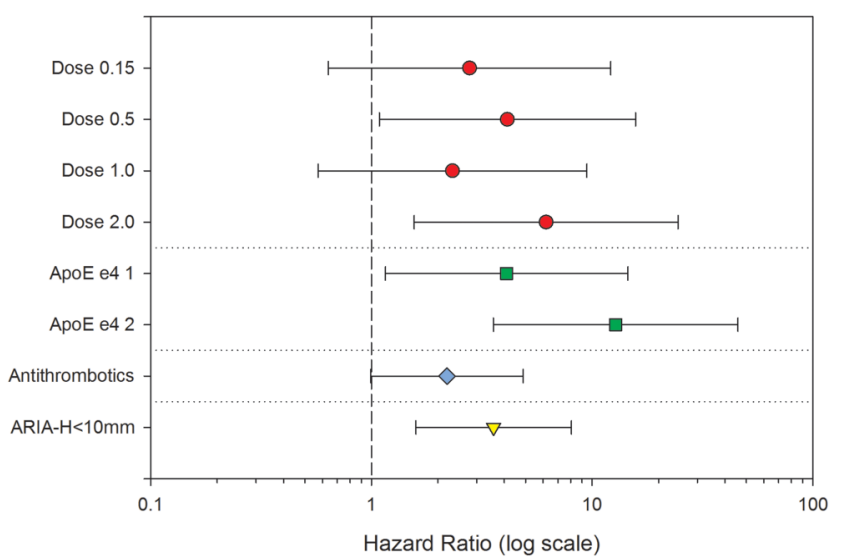

Figure 3 HRs for incident amyloid-related imaging abnormalities-haemosiderin (ARIA-H) $<10 \mathrm{~m}$ from the multivariate proportional hazards model, log scale. 
Table 3 Crude incidence rates and $95 \%$ Cls for ARIA-H $<10 \mathrm{~mm}$ among those receiving bapineuzumab or placebo by the interval time from initiation of therapy

\begin{tabular}{|c|c|c|c|c|c|c|}
\hline \multirow[b]{2}{*}{ Interval } & \multirow[b]{2}{*}{ Rate/100 person-years } & \multicolumn{2}{|c|}{ Bapineuzumab } & \multirow[b]{2}{*}{ Rate/100 person-years } & \multicolumn{2}{|l|}{ Placebo } \\
\hline & & LL $95 \% \mathrm{Cl}$ & UL $95 \% \mathrm{Cl}$ & & LL $95 \% \mathrm{Cl}$ & UL $95 \% \mathrm{Cl}$ \\
\hline$\leq 9$ months & 12.20 & 7.65 & 19.90 & 4.38 & 1.50 & 12.11 \\
\hline$>9$ months & 6.80 & 3.84 & 11.77 & 0.0 & 0.0 & 7.24 \\
\hline Total & 9.22 & 6.41 & 13.08 & 2.55 & 0.87 & 7.23 \\
\hline
\end{tabular}

ARIA-H, amyloid-related imaging abnormalities-haemosiderin; LL, lower limit; UL, upper limit.

APOE $\varepsilon 4$ is a known risk factor for $\mathrm{CAA}$ and $\mathrm{mHs}$, so its identification in this study was expected. ${ }^{17-20}$ Furthermore, APOE $\varepsilon 4$ is associated with increased cerebral amyloid burden. $^{21}$ The limited number of haemorrhagic events precluded an evaluation of a relationship between haemorrhage and either the occurrence of ARIA-H or bapineuzumab. In addition, the apparent transient nature of the risk, a period of increased risk followed by a period without an increased risk, provides an indication of the potential for different mechanisms between the mHs observed in CAA and the ARIA-H observed with disease-modifying therapy.

Murine models of AD treated with anti-a $\beta$ immunotherapy demonstrated increased microhaemorrhages, $<0.2 \mathrm{~mm}$, particularly associated with amyloid-laden vessels. ${ }^{22}$ As the vascular amyloid was removed, the vessel walls appeared to be remodelled with an increased, albeit transient, risk for microhaemorrhages. ${ }^{10}$ Neuropathological studies following immunisation suggested a transient increase in CAA severity with associated increased risk for microhaemorrhages. ${ }^{23}$

The results of this study did not provide sufficient data to determine if an increase in the number of ARIA-H $<10 \mathrm{~mm}$ is associated with ARIA-E overall or with ARIA-E that is symptomatic. The proportion of participants that exhibited any increase was similar, although the proportion was larger (48\% vs 33\%) for those who were not symptomatic.

A challenging observation was the variability in the total number of ARIA-H $<10 \mathrm{~mm}$ over time, with some individuals exhibiting both increases and decreases in the total count. This variability may be due to a multitude of factors including $\mathrm{mHs}$ being out of the MRI slice and confusion with blood vessels. While a standard protocol was utilised in this study, variation is anticipated due to intra-reader variation, changes in MR parameters, such as magnetic field strength, and the participant's orientation, all of which influence the ability to detect specific ARIA-H $<10 \mathrm{~mm}$. However, some of these cases demonstrated a serial decline in numbers of ARIA- $\mathrm{H}<10 \mathrm{~mm}$, indicating that the signal for some lesions had resolved. This is consistent with declines in the number of $\mathrm{mHs}$ observed by others, ${ }^{20} 2425$ which may be the result of resorption of haemosiderin deposition in some cases.

ARIA-H represents a spectrum of MRI findings due to haemosiderin deposition that appears to be related to impaired vascular integrity. The increased risk for ARIA-H associated with APOE $\varepsilon 4$ allele frequency, pre-existing ARIA-H, treatment with bapineuzumab and use of antithrombotic agents provides additional support for this hypothesis of loss of integrity of cerebral vessels due to amyloid burden.

\section{Considerations}

While this study did not show a relationship of ARIA-H $<10 \mathrm{~mm}$ incidence to increasing bapineuzumab dose, the lack of an association should not be interpreted as no relationship. The number of participants who were assigned to the highest dose of bapineuzumab was limited, and overall those participants had a shorter exposure to the study drug than did participants in other dose groups. Furthermore, some of the participants who were initially in the higher dose groups underwent dose titration (dose reduction) due to ARIA-E, and others had their dose reduced when the highest dose level was eliminated by protocol amendment.

Co-location of incident ARIA-H $<10 \mathrm{~mm}$ and ARIA-E was defined as both being located within one of five regions of either hemisphere or the posterior fossa and not necessarily in the identical location. For many of the cases in which ARIA-E

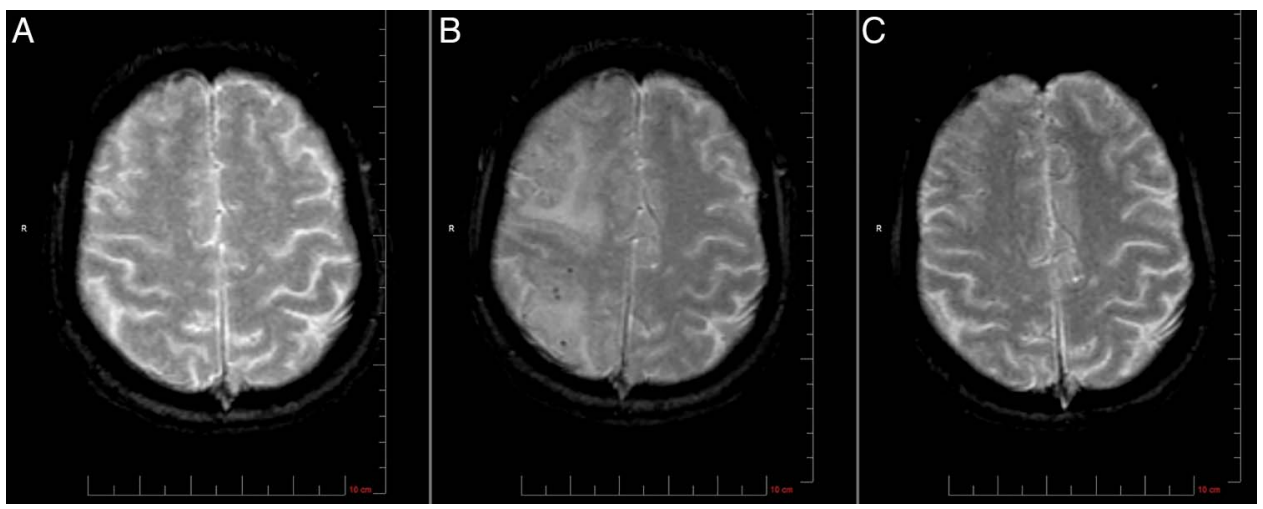

Figure 4 Example of changes in amyloid-related imaging abnormalities-haemosiderin (ARIA-H) seen on T2*/GRE MRI in an APOE $\varepsilon 4$ homozygote patient with Alzheimer's dementia. (A) MRI at baseline showed no ARIA-H $<10 \mathrm{~mm}$. (B) At week 13 after one infusion of active therapy, MRI reveals several $\mathrm{mHs}$ (ARIA-H $<10 \mathrm{~mm}$ ); modest cortical hyperintensity for ARIA-E (ARIA-edema) can be seen as 'shine through'. (C) MRI at week 77 shows resolution of both ARIA-H $<10 \mathrm{~mm}$ and ARIA-E. 
and ARIA-H occurred in the same general region, the abnormalities appeared at different times, were accompanied by a finding in another brain region, or both. Since ARIA-E is a transient event and the identification of single ARIA-H $<10 \mathrm{~mm}$ lesions varied across time, the reclassification of all scans with both ARIA-H $<10 \mathrm{~mm}$ and ARIA-E as to whether or not the imaging findings appear in the same location may have a different result.

While the same imaging protocol was utilised for all the clinical trials, some variation was allowed since the scans were primarily for safety assessment. As the ability to detect haemosiderin deposits is dependent on the specifics of the imaging methods including field strength, echo time, voxel size, asymmetric centring of the echo, and slice thickness, ${ }^{9}$ variations in parameters within a single centre may result in changes in the detection of small ARIA-H. The risk factors for new onset ARIA-H $<10 \mathrm{~mm}$ were observed to be related to the number of APOE $\varepsilon 4$ alleles, bapineuzumab treatment, presence of ARIA $<10 \mathrm{~mm}$ at baseline and use of antithrombotic medication at baseline. As this is the first evaluation of the risk of ARIA $<10 \mathrm{~mm}$ among participants treated with an amyloid mobilising therapy, these factors should be confirmed in other settings. Additional risk factors may have not been identified due to false-negative results or the relatively homogeneous population.

An intriguing observation is that the increased rate for ARIA-H $<10 \mathrm{~mm}$ following initiation of bapineuzumab had a temporal relationship with the risk increased during the initial 6 months and returning to baseline rate thereafter. A similar pattern has been observed with ARIA-E; however, these observations require further evaluation and replication. The variability in the count of ARIA-H $<10 \mathrm{~mm}$ is an intriguing finding and requires further evaluation. The likelihood of a subject experiencing both ARIA-H $<10 \mathrm{~mm}$ and ARIA-E with bapineuzumab appears to be greater than one would expect based on the incidence of each factor independently. However, their co-location within the same general brain region did not appear to be greater than that expected by chance alone. Failure to detect a topographic relationship between ARIA-E and ARIA-H may be influenced by the temporal sampling by MRI, which was conducted at fixed and finite intervals, so that the MR observations of ARIA-E and ARIA-H may not reflect their onset.

\author{
Author affiliations \\ ${ }^{1} J a n s s e n$ Research \& Development, South San Francisco, California, USA \\ ${ }^{2}$ California Pacific Medical Center, San Francisco, California, USA \\ ${ }^{3}$ Synarc, Newark, California, USA \\ ${ }^{4}$ Department of Radiology, Image Analysis Centre, VU University Medical Center, \\ Amsterdam, The Netherlands \\ ${ }^{5}$ Department of Diagnostic Radiology, VU University Medical Center, Amsterdam, \\ The Netherlands \\ ${ }^{6}$ NeuroRx Research, Montreal, Canada \\ ${ }^{7}$ Mayo Clinic, Rochester, Minnesota, USA \\ ${ }^{8}$ Janssen Alzheimer Immunotherapy R\&D, South San Francisco, California, USA \\ ${ }^{9}$ BioMarin, San Rafael, California, USA
}

Acknowledgements The authors would like to acknowledge the participants for their time and effort during the course of these clinical trials. Additionally, they would like to recognise the investigators and their staff during the study.

Contributors HMA and JB contributed to the design of the MRI substudy, data analysis, interpretation of results and drafting of the manuscript. FB, CJ, NK, EL and HRB contributed to the interpretation of results and drafting of the manuscript. DT and DM contributed to the acquisition of the data and interpretation of results. KM contributed to the design of the MRI substudy and interpretation of results.

Funding This study was sponsored by Janssen Alzheimer Immunotherapy Research \& Development, LLC, and Pfizer Inc.

Competing interests HMA, HRB, NK and EL are employees of Janssen Research \& Development. JB, DT, DM and FB was a paid consultant to Janssen Alzheimer Immunotherapy Research \& Development, LLC, during the development of this manuscript. KM was an employee of Janssen Alzheimer Immunotherapy at the time this research was conducted. CJ has been a paid consultant to Janssen Alzheimer Immunotherapy Research \& Development, LLC and Eli Lilly.

Ethics approval Individual Institutional Review and Ethics Committee, 33 sties.

Provenance and peer review Not commissioned; externally peer reviewed.

Open Access This is an Open Access article distributed in accordance with the Creative Commons Attribution Non Commercial (CC BY-NC 4.0) license, which permits others to distribute, remix, adapt, build upon this work non-commercially, and license their derivative works on different terms, provided the original work is properly cited and the use is non-commercial. See: http://creativecommons.org/ licenses/by-nc/4.0/

\section{REFERENCES}

1 Jeerakathil T, Wolf PA, Beiser A, et al. Cerebral microbleeds: prevalence and associations with cardiovascular risk factors in the Framingham study. Stroke 2004;35:1831-5.

2 Vernooji MW, van der Lugt A, Ikram MA, et al. Prevalence and risk factors of cerebral microbleeds: the Rotterdam Scan Study. Neurol 2008;70:1208-14.

3 Cordonnier C, van der Flier WM, Sluimer JD, et al. Prevalence and severity of microbleeds in a memory clinic setting. Neurol 2006;66:1356-60.

4 Hanyu H, Tanaka Y, Shimizu S, et al. Cerebral microbleeds in Alzheimer's disease. J Neurol 2003;250:1496-7.

5 Nakata-Kudo Y, Mizuno T, Yamada K, et al. Microbleeds in Alzheimer disease are more related to cerebral amyloid angiopathy than cerebrovascular disease. Dement Geriatr Cogn Disord 2006;22:8-14.

6 Pettersen JA, Sathiyamoorthy G, Gao FQ, et al. Microbleed topography, leukoaraiosis, and cognition in probably Alzheimer disease from the Sunnybrook dementia study. Arch Neurol 2008;65:790-5.

7 Cordonnier C, Al-Shahi Salman R, Wardlaw J. Spontaneous brain microbleeds: systematic review, subgroup analyses and standards for study design. Brain 2007:130:1988-2003.

8 Goos JDC, Teunissen CE, Veerhuis $R$, et al. Microbleeds related to altered amyloidbeta metabolism in Alzheimer's disease. Neurobiol Aging 2012;33:1011.e1-9.

9 Sperling RA, Jack CR Jr, Black SE, et al. Amyloid-related imaging abnormalities in amyloid-modifying therapeutic trials: recommendations from the Alzheimer's Association Research Roundtable Workgroup. Alzheimer Dement 2011;7:367-85.

10 Zago W, Schroeter S, Guido T, et al. Vascular alterations in PDAPP mice after anti-A immunotherapy: implications for amyloid-related imaging abnormalities. Alzheimer Dement 2013;9:S105-15.

11 Rinne JO, Brooks DJ, Rossor MN, et al. 11C-PiB PET assessment of change in fibrillar amyloid-beta load in patients with Alzheimer's disease treated with bapineuzumab: a phase 2, double-blind, placebo-controlled, ascending-dose study. Lancet Neurol 2010;9:363-72.

12 Salloway S, Sperling R, Gilman S, et al. A phase 2 multiple ascending dose trial of bapineuzumab in mild to moderate Alzheimer disease. Neurology 2009;73:2061-70.

13 Gregoire SM, Chaudhary UJ, Brown MM, et al. The Microbleed Anatomical Rating Scale (MARS): reliability of a tool to map brain microbleeds. Neurology 2009;73:1759-66.

14 Wahlund LO, Barkhof F, Fazekas F, et al. A new rating scale for age-related white matter changes applicable to MRI and CT. Stroke 2001;32:1318-22.

15 Tolappanen AM, Lavikainen P, Soloman A, et al. Incidence of stroke in people with Alzheimer disease: a national register-based approach. Neurology 2013;80:353-8.

16 Salloway S, Sperling R, Fox NC, et al. Bapineuzumab 301 and 302 Clinical Trial Investigators. Two phase 3 trials of Bapineuzumab in mild-to-moderate Alzheimer's disease. New Eng J Med 2014;370:322-33.

17 Greenberg SM, Rebeck GW, Vonsattel JPG, et al. Apolipoprotein E epsilon 4 and cerebral hemorrahge associated with amyloid angioapathy. Ann Neurol 1995;38:254-9.

18 Goos JDC, Henneman WJP, Sluimer JD, et al. Incidence of cerebral microbleeds: a longitudinal study in a memory clinic population. Neurol 2010;74:1954-60.

19 Goos JDC, Kester MI, Barkhof F, et al. Patients with Alzheimer disease with multiple microbleeds: relationship with cerebrospinal fluid biomarkers and cognition. Stroke 2009:49:3455-60.

20 Loehrer E, Ikram MA, Akoudad S, et al. Apolipoprotein E genotype influences spatial distribution of microbleeds. Neurobiol Aging 2014;35:899-905.

21 Caselli RJ, Walker D, Sue L, et al. Amyloid load in nondemented brains correlates with APOE $\varepsilon 4$. Neurosci Lett 2010;473:168-71.

22 Pfeifer $\mathrm{M}$, Boncristiano $\mathrm{S}$, Bondolfi $\mathrm{L}$, et al. Cerebral hemorrhage after passive anti-Abeta immunotherapy. Science 2002;298:1379.

23 Boche $D$, Zotova $E$, Weller $R O$, et al. Consequences of $A \beta$ immunisation on the vasculature of human Alzheimer's disease brain. Brain 2008;131:3299-310.

24 Greenberg SM, O'Donnell HC, Schaefer PW, et al. MRI detection of new hemorrhages: potential marker of progression in cerebral amyloid angiopathy. Neurology 1999;53:1135-8.

25 Kirsch W, McAuley G, Holshouser B, et al. Serial susceptibility weighted MRI measures brain iron and microbleeds in dementia. J Alzheimers Dis 2009;17:599-609. 